\title{
Clinical Features, Diagnosis, and Natural History of Drug-Induced Liver Injury
}

\author{
Paul H. Hayashi, MD, MPH ${ }^{1}$ Robert J. Fontana, MD $^{2}$ \\ ${ }^{1}$ Division of Gastroenterology and Hepatology, University of North \\ Carolina, Chapel Hill, North Carolina \\ 2 Division of Gastroenterology, University of Michigan, \\ Ann Arbor, Michigan \\ Address for correspondence Paul H. Hayashi, MD, MPH, Division of \\ Gastroenterology and Hepatology, University of North Carolina, \\ Burnett-Womack Bldg., Room 8011, Chapel Hill, NC 27599-7584 \\ (e-mail: paul_hayashi@med.unc.edu).
}

Semin Liver Dis 2014;34:134-144.

\begin{abstract}
Keywords

- hepatotoxicity

- diagnostic tools

- prognosis

- histology

- acute

- chronic

There are increasing reports of drug-induced liver injury (DILI) leading to clinically significant acute and chronic liver disease in both children and adults. ${ }^{1-4}$ Drug-induced liver injury remains the leading cause of acute liver failure (ALF) in Western countries and the most common reason for removal of approved medications from the marketplace. ${ }^{5,6}$ The lack of objective diagnostic tests, wide range of clinical presentations and idiosyncratic nature of most cases (i.e., independent of drug dose, duration, route of exposure or identifiable host factors), makes DILI a significant challenge for the practicing gastroenterologist. In the last several years, data acquired from several ongoing prospective registries of DILI cases have started to shed more light on the clinical features, diagnosis, and clinical course of DILI.
\end{abstract}

Patients with idiosyncratic drug-induced liver injury (DILI) can pose substantial diagnostic, prognostic, and therapeutic challenges to the practicing gastroenterologist. The presentation of DILI may vary from asymptomatic liver enzyme elevation to acute liver failure. Although most DILI resolves following drug discontinuation, up to $20 \%$ of patients progress to chronic DILI further challenging the clinicians diagnostic and management skills. Also, some medications can lead to advanced fibrosis, encephalopathy, and portal hypertension without significant elevation in liver enzymes during exposure. Finally, there are no objective tests to definitively diagnose DILI. Although causality assessment instruments are available, none are widely accepted or used in clinical practice. Therefore, the diagnosis of DILI depends on thorough and accurate history taking, follow-up of the patient's clinical course and excluding more common causes of liver injury. In this review, we discuss the variable clinical presentations, course, and diagnostic methods used to establish a diagnosis and prognosis in DILI.

\section{Symptoms and Signs of Drug-Induced Liver Injury}

The initial symptoms and signs of DILI are often nonspecific (e.g., fatigue, nausea, and abdominal pain). In the U.S. Drug
Induced Liver Injury Network (DILIN) registry of over 1,200 consecutive cases, nausea was present in $60 \%$ and abdominal pain in $42 \%$ (Robert Fontana, personal communication). The onset of clinical symptoms can be important in determining the latency of a possible DILI episode. Liver-specific symptoms and signs (e.g., pruritus, jaundice, ascites, and encephalopathy) are usually only present in patients with more severe DILI. In the DILIN and Spanish registries, $\sim 70 \%$ of patients were jaundiced at presentation and $51 \%$ had pruritus. Jaundice in the setting of an acute hepatocellular injury, is associated with a mortality of $10 \%$, often referred to as Hy's Law after the late Hy Zimmerman. 1,2,7,8 Ascites and encephalopathy are well-known ominous signs of hepatic failure. ${ }^{9}$ Drug-induced liver injury remains the overall leading cause of acute liver failure (ALF) in the U.S. and idiosyncratic DILI is the second leading cause among cases where an etiology is identified. ${ }^{10}$ The leading agents causing ALF are antituberculosis agents (isoniazid), antiepileptics (phenytoin, valproate), and antibiotics (ketoconazole, nitrofurantoin), followed by herbal and dietary supplements (HDS). ${ }^{10,11}$
Issue Theme Drug-Induced Liver Injury; Guest Editors, Naga Chalasani, MD, and Paul H. Hayashi, MD, MPH
Copyright @ 2014 by Thieme Medical Publishers, Inc., 333 Seventh Avenue, New York, NY 10001, USA. Tel: +1(212) 584-4662.
DOI http://dx.doi.org/ 10.1055/s-0034-1375955. ISSN 0272-8087. 
Drugs and HDS products may also cause subclinical liver injury.For example, isoniazid therapy for latent TB will cause mild serumalanine aminotransferase (ALT) elevation in up to 20 to30\% of treated patients. Many of these will develop tolerance and experience a decline in ALT levels despite continued INH use. $^{12-14}$ The heparin compounds can cause asymptomatic serum ALT elevations as well. A recent study in healthy volunteers showed that most will have some elevation in serum ALT levels and many had elevations greater than 3 to 5 times the upper limit of normal. ${ }^{15}$ These elevations occur with all the heparin compounds even when delivered subcutaneously and resolve with continued therapy. ${ }^{16}$ The combination of human immunodeficiency virus (HIV) antiretrovirals, atazanavir, and ritonavir can also cause selflimited unconjugated hyperbilirubinemia in up to $44 \%$ of treated patients that resolves with drug discontinuation. ${ }^{17}$

Immunoallergic features may be a prominent feature in some DILI patients and at least one immunologic feature was present in $23 \%$ of the DILIN patients. ${ }^{2}$ Certain agents such as allopurinol, sulfamethoxazole/trimethoprim, vancomycin, and phenytoin ${ }^{18-21}$ frequently present with prominent immunoallergic features such as rash, fever, serositis, eosinophilia, bone marrow suppression, and multiorgan involvement (DRESS). In general, rechallenge in such cases will lead to a more rapid recurrence presumably due to immunologic memory of $\mathrm{T}$ and $\mathrm{B}$ cells. Other medications such as nitrofurantoin, minocycline, and $\alpha$-methyldopa may cause an immune-mediated injury that is indistinguishable from sporadic autoimmune hepatitis. Autoimmune markers (antinuclear and antismooth muscle antibodies) may be markedly positive and histology may look identical to autoimmune hepatitis. The latencies can be quite long (months to years) and confidently distinguishing DILI from autoimmune hepatitis $(\mathrm{AIH})$ often depends on resolution with medication discontinuance and lack of need for prolonged immunosuppressive therapy. ${ }^{22}$ Biologics including anti-TNF agents used to treat inflammatory bowel disease can also lead to severe acute liver injury with autoimmune hepatitis-like features. ${ }^{23}$

\section{Atypical Clinical Presentations}

Drug-induced liver injury can occasionally present with only modest or no elevations in liver biochemistries. Chronic use of methotrexate is probably the most well-known example. Serum aminotransaminase elevations are typically mild, yet a steatotic liver injury with fibrosis can occur over months to years of therapy. ${ }^{24}$ The risk of cirrhosis was probably exaggerated in early reports due to confounding from concomitant alcohol consumption as well as underlying nonalcoholic fatty liver disease. Nevertheless, the potential for chronic liver injury in up to 5 to $10 \%$ of treated patients is widely accepted and guidelines from both dermatology ${ }^{25}$ and rheumatolo$\mathrm{gy}^{26,27}$ professional societies recommend monitoring of serum aminotransferase levels with periodic liver biopsy depending on the levels, patient risk factors, and cumulative drug exposure.

Hepatotoxicity from the antiepileptic, valproate is noteworthy for its three distinct presentations (- Table 1). ${ }^{28,29}$ It may present acutely with jaundice, anorexia, and encepha- lopathy. Oddly, liver enzyme elevations may be modest in comparison to other injuries presenting with such severe hepatic dysfunction. Valproate hepatotoxicity can also present abruptly with a Reye's-like syndrome typically in children. Here again, liver biochemistry abnormalities are often modest and overshadowed by the neurologic complaints of anorexia, lethargy, cerebral edema, and coma. Lastly, valproate can cause a hyperammonemic encephalopathy without overt liver injury. ${ }^{30}$ The reasons for this odd array of presentations lie in valproate's mitochondrial toxicity. Microvesicular steatosis is seen on liver biopsy and carnitine depletion is felt to play a role in the pathophysiology. Mutations in the gamma polymerase gene that codes for the predominant DNA polymerase in mitochondria may influence patient susceptiblity. ${ }^{31}$

Patients with DILI may also rarely present withnoncirrhotic portal hypertension and associated variceal bleeding and/or ascites, but preserved hepatic synthetic function. Nodular regenerative hyperplasia (NRH) may be present on needle biopsy, but other times histology is unrevealing. Several medications including oral contraceptives, antineoplastics, and immunosuppressives have been implicated. Due to the indolent development of portal hypertension from stellate cell stimulation and liver regeneration, latency periods can be long. Azathioprine, which has been associated with $\mathrm{NRH}$, remains a mainstay treatment for inflammatory bowel disease and autoimmune hepatitis. Oxaliplatin is commonly used for stage III colon cancer and has also recently been associated with significant portal hypertension in the absence of overt liver inflammation or synthetic dysfunction. $^{32,33}$ Both drugs also have been linked to sinusoidal obstructive syndrome.

Sinusoidal obstructive syndrome (SOD) usually presents more abruptly with evidence of portal hypertension and signs of hepatic dysfunction, but liver biochemistries may be only mildly elevated. Sinusoidal obstructive syndrome is typically associated with myeloablative chemotherapy given for hematologic malignancies, but other chemotherapeutic agents given for other diseases have also been implicated. ${ }^{34}$ Sinusoidal obstructive syndrome typically presents with right upperquadrant pain, weight gain, jaundice, and hepatomegaly of varying severity. Ascites may or may not be present. Although drug latency is usually short (i.e., 20-30 days), the diagnosis can be difficult to confidently establish because these patients are often at risk for other causes of liver injury including opportunistic infections, sepsis, ischemia, and exposure to other hepatotoxic medications including antifungals and antibacterials. Furthermore, it may be difficult to distinguish early graft versus host disease from delayed SOD. Two diagnostic criteria have been published for SOD, but still 10-20\% cannot be diagnosed definitely without a biopsy. ${ }^{35,36}$

\section{Diagnostic Evaluation of Suspected Drug- Induced Liver Injury}

Drug-induced liver injury diagnosis depends on obtaining a meticulous history and thoughtful use of diagnostic tests. However, making this effort upfront can save weeks in 
136 Clinical Spectrum and Diagnosis of Drug-Induced Liver Injury Hayashi, Fontana

Table 1 Latency and presentation with commonly implicated drugs that may cause liver injury

\begin{tabular}{|c|c|c|}
\hline Antibiotics & Typical latency* & Typical pattern of injury / identifying features \\
\hline Amoxicillin/clavulanate & $1-4$ wk & $\begin{array}{l}\text { Cholestatic injury, but can be hepatocellular at initial } \\
\text { presentation }\end{array}$ \\
\hline Isoniazid & $1-6 \mathrm{mo}$ & Acute hepatocellular injury similar to acute viral hepatitis \\
\hline Sulfamethoxazole/trimethoprim & $\begin{array}{l}\text { Short to moderate } \\
(<4 \mathrm{wk})\end{array}$ & $\begin{array}{l}\text { Cholestatic injury, but can be hepatocellular; often with } \\
\text { immunoallergic features (e.g., rash, eosinophilia) }\end{array}$ \\
\hline Fluoroquinolones & $\begin{array}{l}\text { Short } \\
(1-14 d)\end{array}$ & Equally hepatocellular, cholestatic or mixed \\
\hline \multicolumn{3}{|l|}{ Nitrofurantoin } \\
\hline Acute form (rare) & Short & Hepatocellular \\
\hline Chronic form & Moderate to Long (mo-y) & $\begin{array}{l}\text { Typically hepatocellular and often identical to autoim- } \\
\text { mune hepatitis }\end{array}$ \\
\hline Minocycline & Moderate to Long & $\begin{array}{l}\text { Hepatocellular and often identical to autoimmune } \\
\text { hepatitis }\end{array}$ \\
\hline \multicolumn{3}{|l|}{ Antiepileptics } \\
\hline Phenytoin & Short to moderate & $\begin{array}{l}\text { Hepatocellular, mixed or cholestatic often with } \\
\text { immunoallergic features (e.g., rash, eosinophilia) }\end{array}$ \\
\hline Carbamazepine & Moderate & $\begin{array}{l}\text { Hepatocellular, mixed or cholestatic often with } \\
\text { immunoallergic features }\end{array}$ \\
\hline Lamictal & Moderate & Hepatocellular often with immunoallergic features \\
\hline \multicolumn{3}{|l|}{ Valproate } \\
\hline Hyperammonia & Moderate to long & Elevated ammonia, encephalopathy \\
\hline Hepatocellular & Moderate to long & Hepatocellular \\
\hline Reye-like syndrome & Short to moderate & $\begin{array}{l}\text { Hepatocellular, acidosis; microvesicular steatosis on } \\
\text { biopsy }\end{array}$ \\
\hline \multicolumn{3}{|l|}{ Analgesics } \\
\hline Nonsteroidal anti-inflammatory agents & Moderate to long & Hepatocellular injury \\
\hline \multicolumn{3}{|l|}{ Immune modulators } \\
\hline Interferon $\beta$ & Moderate to long & Hepatocellular, female predominance \\
\hline \multicolumn{3}{|l|}{ Miscellaneous } \\
\hline Methotrexate (oral) & Long (> 1 y) & Fatty liver, fibrosis \\
\hline Allopurinol & Short to moderate & $\begin{array}{l}\text { Hepatocellular or mixed. Often with immunoallergic } \\
\text { features; granulomas on biopsy }\end{array}$ \\
\hline Amiodarone (oral) & Moderate to long & $\begin{array}{l}\text { Hepatocellular, mixed or cholestatic; macrovesicular } \\
\text { steatosis on biopsy }\end{array}$ \\
\hline Androgen-containing steroids & Moderate to long & $\begin{array}{l}\text { Cholestatic. Can present with peliosis hepatitis, nodular } \\
\text { regenerative hyperplasia or hepatocellular carcinoma }\end{array}$ \\
\hline Inhaled anesthetics & Short & $\begin{array}{l}\text { Hepatocellular. May have immunoallergic } \\
\text { features } \pm \text { fever. }\end{array}$ \\
\hline \multicolumn{3}{|l|}{ Gastrointestinal Medications } \\
\hline Interferon alpha & Moderate & Hepatocellular, autoimmune hepatitis-like \\
\hline Anti-tumor necrosis factor agents & Moderate to long & Hepatocellular. Can have autoimmune hepatitis features \\
\hline Azathioprine & Moderate to long & $\begin{array}{l}\text { Cholestatic or hepatocellular, but can present with portal } \\
\text { hypertension (veno-occlusive disease, nodular } \\
\text { regenerative hyperplasia) }\end{array}$ \\
\hline Macrolides & Short & Hepatocellular, but can be cholestatic \\
\hline Sulfasalazine & Short to moderate & $\begin{array}{l}\text { Mixed, hepatocellular, or cholestatic; often with } \\
\text { immunoallergic features }\end{array}$ \\
\hline Proton pump inhibitors & Short & Hepatocellular; very rare \\
\hline
\end{tabular}

*Short $=3-30$ days, Moderate $=30-90$ days, Long $>90$ days unless otherwise specified. 
diagnostic evaluation, decrease morbidity, and avoid unnecessary tests. Overall, the assessment focuses on four major areas: (1) timing (exposure or latency; recovery or dechallenge), (2) pattern of liver biochemistries at presentation, (3) hepatotoxicity profile of suspect agent, and (4) exclusion of competing causes. Judicious use of blood tests and liver imaging are necessary, but liver biopsy, while often helpful, is not mandatory. Drug-induced liver injury assessment has been organized into diagnostic scoring systems ${ }^{37-39}$ that are useful in organizing data into a categorical framework. However, they are not widely used in practice due to lack of proven reliability and accuracy. Others have published more complete lists of necessary clinical data that can serve as a checklist for the clinician (- Table 2). ${ }^{40,41}$

The importance of getting accurate timing of medication start and stop dates (exposure), onset of symptoms, or liver biochemistry abnormalities (latency) and liver recovery (dechallenge) cannot be overemphasized. Such timing information is the initial parameter for all diagnostic algorithms ${ }^{37,39}$ because inaccurate exposure data will undermine any final diagnosis. For prescription drugs, contacting the patient's pharmacy can be invaluable in defining exposure and completeness of all medications taken. ${ }^{42}$ Nowadays, getting a complete medication list also includes asking patients about herbal and dietary supplement (HDS) use; supplements are taken by over $50 \%$ of the U.S. population. ${ }^{43}$ Recent data suggest HDS hepatotoxicity attributed to body building supplements, weight loss products, and other formulations containing various amounts of potentially hepatotoxic ingredients (e.g., catechins) is on the rise in the United States. ${ }^{44,45}$ Determining onset of signs or symptoms is particularly challenging because a patient's memory can be

Table 2 Minimum elements of a diagnostic evaluation in patients with suspected drug-induced liver injury

\begin{tabular}{|c|c|}
\hline Element & Comments \\
\hline Gender & Pertinent for some competing disorders (e.g., PBC) \\
\hline Age & Pertinent for some competing disorders (e.g., HEV) \\
\hline Race/ethnicity & $\begin{array}{l}\text { Pertinent for some competing disorders (e.g., sarcoidosis, sickle cell-related } \\
\text { liver injury, oriental sclerosing cholangitis) }\end{array}$ \\
\hline Indication for suspect drug or HDS use & $\begin{array}{l}\text { May have underlying liver disease (hypoglycemic agents in diabetics, weight- } \\
\text { loss products in obese, etc.) }\end{array}$ \\
\hline Concomitant diseases & $\begin{array}{l}\text { Particularly pertinent disorders may include sepsis, heart failure, hypotension } \\
\text { episodes, recent general anesthesia, parenteral nutrition, and cancer }\end{array}$ \\
\hline Presence of rechallenge & Give timing of rechallenge if done \\
\hline History of other drug reactions & Certain cross reactivities may exist (e.g., antiepileptics) \\
\hline History of other liver disorders & $\begin{array}{l}\text { Chronic viral hepatitis, NAFLD, hemochromatosis, alcoholic liver disease, PSC, } \\
\text { PBC, liver cancer }\end{array}$ \\
\hline History of alcohol use & $\begin{array}{l}\text { Past versus present; estimated grams per day; sporadic versus binge drinking } \\
\text { versus regular (daily or weekly) }\end{array}$ \\
\hline Exposure time & Start and stop dates or total number of days, weeks, or months taken. \\
\hline Symptoms \& signs & $\begin{array}{l}\text { Presence or absence, time of onset, type (fatigue, weakness, abdominal pain, } \\
\text { nausea, dark urine, icterus, jaundice, pruritus, fever, rash) }\end{array}$ \\
\hline Physical findings & Fever, rash, hepatic tenderness, signs of chronic liver disease \\
\hline Medications \& HDS products & $\begin{array}{l}\text { Complete list of medications or HDS products with particular attention to } \\
\text { those started in the previous 3-6 mo }\end{array}$ \\
\hline Laboratory results & $\begin{array}{l}\text { Day of first abnormal liver biochemistry; liver biochemistries, eosinophil counts } \\
\text { at presentation }\end{array}$ \\
\hline Viral hepatitis serologies & Anti-HAV IgM, HBsAg, anti-HBc IgM, anti-HCV, HCV RNA \\
\hline Autoimmune hepatitis serologies & ANA, antismooth muscle antibody, IgG level \\
\hline Imaging & US \pm Doppler, $\mathrm{CT}$, or MRI \pm MRCP \\
\hline Histology if available & $\begin{array}{l}\text { Timing of biopsy in relation to enzyme elevation and drug-induced liver injury } \\
\text { onset }\end{array}$ \\
\hline Washout (dechallenge) data & Follow-up liver biochemistries over 3-6 mo after drug discontinuation \\
\hline Clinical outcome & Resolution versus chronicity, transplant, death, and timing of each \\
\hline
\end{tabular}

Abbreviations: ANA, antinuclear antibody; $C T$, computerized tomography; HAV, hepatitis A virus; $\mathrm{HBc}$, hepatitis B core antigen; $\mathrm{HBs}$, hepatitis B surface antigen; HCV, hepatitis C virus; HDS, herbal or dietary supplement; HEV, hepatitis E virus; Ig, immunoglobulin; MRCP, magnetic resonance cholangiopancreatography; MRI, magnetic resonance imaging; NAFLD, nonalcoholic fatty liver disease; PBC, primary biliary cirrhosis; PSC, primary sclerosing cholangitis; RNA, ribonucleic acid; US, ultrasound.

Source: Modified from Agarwal VK et al. ${ }^{40}$ 
vague and subject to recall bias. Interviewing family and friends may be necessary, and recall cues used in epidemiology research can be helpful. ${ }^{46,47}$

The pattern of liver biochemistry elevations at presentation issecond only to a good history in diagnostic importance. Elevationsare often categorized by the $R$ value $R=$ [ALT value/ALT upper limit of normal $] \div[$ alkaline phosphatase (AP) value/AP upper limit of normal]). ${ }^{48} R$ values of $>5$ are considered hepatocellular, $<2$ cholestatic, and 2-5 mixed. These cutoffs are somewhat arbitrary and $R$ values can also change as the injury progresses, particularly from hepatocellular to cholestatic pattern over time. ${ }^{38}$ Nevertheless, they serve as a useful way to focus a diagnostic evaluation on particular hepatotoxic agents and competing diagnoses.

\section{Likelihood of Liver Injury from a Drug}

Knowledge of the likelihood that a given drug can cause hepatotoxicity is important when assessing a patient with possible DILI. Overall, antibiotics and antiepileptics are most commonly reported accounting for up to $60 \%$ of DILI. ${ }^{1}$ Therefore, the appearance of either of these two classes of agents on a medication list should heighten one's suspicion for DILI. On the other hand, antihypertensive and diabetic medications are less commonly reported. ${ }^{1}$ Certain offenders have signature presentations (e.g., amoxicillin-clavulanate, isoniazid, phenytoin), and - Table 1 lists the most notorious as well as commonly prescribed agents including those often prescribed by gastroenterologists. For example, isoniazid injury is virtually always hepatocellular and fluoroquinolone injury typically has a very short latency. Idiosyncratic DILI inherently offers few generalizations across all medications, but a recent study suggests drugs given in daily doses exceeding $100 \mathrm{mg} / \mathrm{d}$ and those that are more lipophilic may be more likely to cause hepatotoxicity. ${ }^{49,50}$

Staying abreast of less well-known or newly reported agents associated with DILI is more difficult with the Food and Drug Administration (FDA) having approved an average of 90 drugs per year from 2007-2011 alone. ${ }^{51}$ Published DILI cases are spread across subspecialty, toxicology, pharmacology, and gastroenterology journals. Recently, the National Institute of Diabetes and Digestive and Kidney Diseases (NIDDK) and the National Library of Medicine (NLM) launched LiverTox (http://www.livertox.nih.gov/), a free online resource that is updated on a regular basis. ${ }^{52,53}$ Over 650 medications are included on the website and this number continues to grow. Each medication is presented in a concise and clinically useful manner. References are robust and linked to the NLM. This website has quickly become a mainstay tool to the clinician and researcher alike with over 30,000 visits per month. There are plans to expand the LiverTox website to include all marketed prescription drugs and some HDS products, as well as provide a computerized causality assessment instrument to assist in DILI diagnosis.

\section{Competing Causes of Liver Injury}

Searching for more common competing diagnoses of liver injury based upon the laboratory profile at presentation is also important. Hepatocellular injuries prompt suspicions of viral hepatitis, ischemia, and autoimmune hepatitis. A detailed alcohol history is critical when the transaminase pattern (modest elevation; aspartate aminotransferase [AST] $>2 \mathrm{x}$ ALT) is consistent with alcoholic hepatitis. A rapid rise and fall of serum aminotransaminase levels are hallmarks for ischemic injury. Autoimmune hepatitis (AIH) is often one of the more difficult competing diagnosis to eliminate because like DILI there is no single objective diagnostic test for $\mathrm{AIH}^{54}$ Budd-Chiari syndrome can also present with acute hepatocellular injury and should be pursued with appropriate imaging studies (e.g., Doppler ultrasound, computed tomography or magnetic resonance imaging).

Such diagnostic evaluation of hepatocellular enzyme elevation is well known to the gastroenterologist. However, there are some noteworthy diagnoses that masquerade as DILI. Even though 10,000 new infections occur in the United States annually, acute hepatitis $C$ is often overlooked because gastroenterologists are more accustomed to seeing the indolent chronic phase of infection, and diagnostic test results are variable in acute infection. Very early in infection, hepatitis $C$ virus (HCV) antibody can be negative, and HCV RNA testing may be necessary. Detectable HCV RNA without antibody is consistent with acute infection. Seroconversion in the following 4 to 12 weeks with or without loss of HCV RNA would be strong evidence for acute infection, particularly if a recent risk factor were identified. Ultimately, repeat history taking for hepatitis $\mathrm{C}$ risk factors and retesting of HCV antibody and RNA in 4 to 12 weeks should be done, but are often forgotten. Pursuing the diagnosis of acute hepatitis $C$ takes on added importance as we enter an era of more tolerable and curative therapies.

Cytomegalovirus (CMV) and Epstein Barr virus (EBV) hepatitis are uncommon in the immunocompetent host, ${ }^{55}$ but hepatocellular liver enzyme elevation in systemic CMV infection is often seen. ${ }^{56,57}$ Herpes simplex virus (HSV) hepatitis patients are frequently younger, with high fever and can have quite severe or fatal liver injury. ${ }^{58}$ Immunocompromised patients are more at risk, but cases in the immunocompetent are described. ${ }^{59}$ All three have acute serologic panels as well as polymerase chain reaction (PCR) testing available. No studies have looked systematically at how often suspected DILI cases are actually HSV, EBV, or CMV hepatitis. Therefore, these viral infections should be considered in cases with suggestive symptoms (e.g., fever, lymphadenopathy, splenomegaly, herpetic lesions). For all viral infections, antibody testing may be less sensitive in the immunocompromised host and nucleic acid testing should be done.

Hepatitis E is uncommon in North America and Western Europe, but has been documented to masquerade as DILI. Dalton and colleagues suggested a $12 \%$ acute hepatitis $\mathrm{E}$ rate in 47 cases thought to be DILI from the United Kingdom and New Zealand based on hepatitis E virus (HEV) IgG and IgM serologies. ${ }^{60}$ The DILIN retrospectively searched its registry for evidence of acute hepatitis E in 318 cases. All nine cases with positive HEV serologies were tested for HEV RNA, and re-evaluated by the DILIN group for likelihood of DILI versus HEV. Seven (2\%) were felt to be more likely acute hepatitis E 
than DILI after re-evaluation. ${ }^{61}$ These cases of unsuspected acute HEV were predominantly in men over 50 years of age in both studies. Although the zoonotic spread of HEV from pigs, boar, and deer is postulated, a strong epidemiologic link is lacking. ${ }^{62}$ Outbreaks associated with travel to endemic areas (e.g., Southeast Asia, Asian subcontinent, Africa, and Mexico) are seen. Currently, tests for anti-HEV IgG and IgM levels are commercially available, but not FDA approved. In addition, testing for HEV RNA by PCR is not available in the United States. Therefore, routinely testing for anti-HEV cannot be recommended at this time, but may be considered if there is a potential exposure history (e.g., recent travel to endemic regions).

Though rare, Wilson disease is often considered as a competing diagnosis during the workup for acute hepatocellular injury particularly when acute liver failure (ALF) is present. Diagnostic guidelines for Wilson disease are available, ${ }^{63}$ but if ALF is present, then the ratios of AP:bilirubin $<4$ and AST:ALT $>2.2$ have shown better diagnostic accuracy. ${ }^{64}$

Cholestatic injuries prompt concerns for biliary problems such as choledocholithiasis, pancreaticobiliary tumors, strictures, and infiltrating cancer. Evaluation for these disorders is commonplace for the gastroenterologist. Guidelines for the role of endoscopic retrograde cholangiography (ERC) and endoscopic ultrasound (EUS) in the evaluation and treatment of choledocholithiasis were published in 2010 and are driven by serum bilirubin levels $>1.8$ to $4.0 \mathrm{mg} / \mathrm{dL}$ and ultrasound imaging. ${ }^{65}$ However, these guidelines are specifically for patients with "symptomatic cholelithiasis." In contrast to stone obstruction, cholestatic DILI is often more insidious in onset with pruritus, fatigue, nonspecific abdominal complaints, or no symptoms at all. Therefore, caution should be taken in applying the American Society for Gastrointestinal Endoscopy (ASGE) algorithm for early ERC in the absence of clinical symptoms or ductal dilation on imaging, even when the bilirubin is $>4 \mathrm{mg} / \mathrm{dL}$, a "very strong" predictor for bile duct stone when typical cholelithiasis symptoms are present. Indeed, the absence of duct dilation on ultrasound carries a 95\% negative predictive value for choledocholithiasis especially with a bilirubin $>5$ to $6 \mathrm{mg} / \mathrm{dL}$. ${ }^{66}$ First-time presentations of primary biliary cirrhosis ( $\mathrm{PBC}$ ) and primary sclerosing cholangitis (PSC) are also familiar to the gastroenterologist and diagnostic guidelines are available. ${ }^{67,68}$

The differential and diagnostic evaluation of patients with an acute "mixed" liver injury pattern is broader. Such mixed pattern liver biochemistries can be particularly challenging because transition from predominantly hepatocellular to cholestatic injury can occur. A patient may present late with cholestatic enzyme elevation and the prior elevation of transaminases was missed. Here the latency between symptom onset and first testing of liver enzymes may be a helpful clue.

\section{Liver Biopsy}

A diagnosis of DILI does not require a liver biopsy, but a biopsycan be helpful in confirming a clinical suspicion of DILI and helping to exclude competing etiologies. ${ }^{1}$ Some histologic findings may be quite suggestive of possible DILI ( - Table 3 ) and textbook descriptions of these are available. ${ }^{69,70}$ Kleiner et al recently catalogued the histologic findings from 249 consecutive DILIN cases and found most (83\%) fall into six major categories of injury (acute hepatitis, chronic hepatitis, acute cholestasis, chronic cholestasis, zonal necrosis, and cholestatic hepatitis). ${ }^{71}$ Interestingly, the correlation with the $R$ value was not very strong with significant overlap of $R$ values across the histologic categories. However, certain histologic findings such as necrosis, fibrosis, and microvesicular steatosis were associated with worse outcomes, whereas granulomas and

Table 3 Clinical phenotypes of drug-induced liver injury, histologic features, and exemplary agents

\begin{tabular}{|l|l|l|}
\hline Phenotype & Histological features & Example agents \\
\hline Acute fatty liver with lactic acidosis & $\begin{array}{l}\text { Microvesicular hepatic steatosis } \pm \text { other tissue } \\
\text { involvement }\end{array}$ & Didanosine, Fialuridine, Valproate \\
\hline Acute hepatic necrosis & Collapse and necrosis of liver parenchyma & $\begin{array}{l}\text { Isoniazid } \\
\text { Niacin }\end{array}$ \\
\hline Autoimmune-like hepatitis & $\begin{array}{l}\text { Plasma cells \& interface hepatitis with detectable } \\
\text { autoantibodies }\end{array}$ & Nitrofurantoin, Minocycline \\
\hline Bland cholestasis & Balloon hepatocytes with minimal inflammation & Anabolic steroids \\
\hline Cholestatic hepatitis & $\begin{array}{l}\text { Balloon hepatocytes with inflammation, predomi- } \\
\text { nance of serum alkaline phosphate elevation } \\
\text { (phenytoin, amoxicillin-clavulanate) }\end{array}$ & Phenytoin, Amoxicillin-clavulanate \\
\hline Fibrosis/ cirrhosis & Hepatic collagenization with minimal inflammation & Methotrexate, Amiodarone \\
\hline Immunoallergic hepatitis & Eosinophilic infiltrate & Trimethoprim-sulfamethoxazole \\
\hline Nodular regeneration & Micro- or macroscopic liver nodules & Azathioprine, Oxaliplatin \\
\hline Nonalcoholic fatty liver & $\begin{array}{l}\text { Macro- and microsteatosis, hepatocyte ballooning } \\
\text { and periportal inflammation }\end{array}$ & Tamoxifen \\
\hline Sinusoidal obstruction syndrome & Inflammation with obliteration of central veins & Busulfan \\
\hline Vanishing bile duct syndrome & Paucity of interlobular bile ducts & Sulfonamides, Beta-lactams \\
\hline
\end{tabular}


eosinophilic infiltrates were associated with better outcomes as suggested in prior studies. ${ }^{71-73}$

A biopsy may be mandatory when autoimmune hepatitis (AIH) is a strong competing possibility because diagnostic criteria for AIH include histology. ${ }^{74}$ Commitment to immunosuppressive therapy for $\mathrm{AIH}$ is often long term and carries risks and side effects. ${ }^{75,76}$ In general, persistence of liver biochemistry abnormalities also warrants a liver biopsy because the majority of DILI cases show improvement in liver biochemistries after drug discontinuation. Therefore, persistence of biochemical abnormalities strengthens the possibility of a non-DILI diagnosis that may be elucidated by a biopsy. The decision on when to obtain a liver biopsy is more art than science. One algorithm considers lack of a 50\% drop in the difference between ALT peak and upper limit of normal (ULN) 30 days after stopping the suspected agent as weakening a DILI diagnosis significantly. ${ }^{37}$ Another puts the cutoff at 60 days. $^{39}$ For cholestatic liver injury, a lack of significant drop in AP or bilirubin levels ( $>50 \%$ drop in peak-ULN or drop to $<$ twice ULN) at 180 days is considered significant. There are no prospective studies examining the yield of biopsy based on these cutoffs. However, considering a biopsy at 60 days for hepatocellular and 180 days for cholestatic enzyme patterns is reasonable. Earlier biopsy may be justified for continued rise in liver biochemistries particularly when any signs of liver failure arise.

Occasionally, a liver biopsy may be necessary for continued use or contemplated rechallenge with an implicated medication such as a chemotherapeutic drug for advanced malignancy. Guidelines for when to obtain a liver biopsy with chronic methotrexate use are published. ${ }^{25,26}$ The Roenigk Classification System is the recognized histologic grading system for methotrexate injury. ${ }^{77}$

\section{Diagnostic Instruments: Roussel Uclaf Causality Assessment Model}

There are two DILI specific scoring diagnostic algorithms, 37,39 but only the Roussel Uclaf Causality Assessment Model (RUCAM) has found traction clinically. The RUCAM was intended for use at the bedside or in clinic, and yields a summed score from -10 to 14 , higher scores indicating higher likelihood of DILI. ${ }^{37}$ Scores are grouped into likelihood levels of "excluded" (score $\leq 0$ ), "unlikely (1-2), "possible" (3-5), "probable" (6-8), and "highly probable" (>8). This scoring system is divided into hepatocellular injuries and cholestatic or mixed injuries. Points are given or taken away based on timing, dechallenge, risk factors for DILI, competing medications, competing diagnoses, and rechallenge information (-Table 4). Although simple in concept, ambiguity on how to score certain sections hinders its use. Alcohol use is a risk factor, but not clearly defined. Points are given to a "known hepatotoxin," but precise definition of such is unclear. These areas of ambiguity probably contribute to RUCAM's suboptimal retest reliability (reliability coefficient of 0.51 , upper $95 \%$ confidence limit 0.76). ${ }^{78}$

Validation is difficult without a gold standard for diagnosing DILI, but was attempted using rechallenge and competing hepatotoxin cases as positive and negative controls, respec- tively. ${ }^{38}$ But rechallenge and competing agents are part of the RUCAM algorithm itself hindering the validation analysis. The RUCAM has been compared with the DILIN expert opinion process. ${ }^{79}$ Three DILIN hepatologists, using a set protocol, come to a consensus of DILI likelihood. ${ }^{80}$ One of five categories similar to the RUCAM is assigned. RUCAM and DILIN concordance across the five categories was modest by Spearman's coefficient $(0.42, p<0.05)$, but agreement for discerning at least "probable" versus "possible" was 69\% with positive (PPV) and negative (NPV) predictive values of $95 \%$ and $23 \%$, respectively. If a clinician were merely interested in whether DILI was at least "possible," the RUCAM agreed with expert opinion 94\% of the time with PPV and NPV of 98\% and $37 \%$, respectively. Hence, the RUCAM did well in identifying the possibility of DILI, but it could not rule it out.

Although the RUCAM is not a standalone diagnostic instrument, it can be an adjunct to expert opinion. Perhaps its greatest utility is in providing a framework upon which the clinician can organize history taking and tests. It reminds the clinician of the important areas of a DILI history and requires precision on exposure times and latency. ${ }^{81}$

\section{Natural History}

The low incidence and heterogeneity of DILI makes research into its natural history difficult, but large registries and population-based studies are beginning to clarify this issue. In Iceland, the crude overall annual incidence of idiosyncratic DILI was 19.1 case per 100,000 population, which is similar to the rate reported previously in northern France., ${ }^{3,4}$ Three registries from Sweden, Spain, and the United States totaling over 1,500 patients reported 6 to $9 \%$ having a severe outcome of death or need for liver transplantation within 6 months. ${ }^{1,2,8}$ Risk of such early adverse outcome was highest in those with acute hepatocellular injury (7-13\%) and lowest with a mixed pattern (2\%). However, within these overall severe outcome rates there was wide variation between drugs. In the Swedish registry, both isoniazid and halothane cases of hepatocellular injury had $40 \%$ rates of death or transplantation, whereas no such severe outcomes were seen with erythromycin. ${ }^{8}$ Across all three studies, elevated bilirubin at presentation was associated with early severe outcome. Therefore, patients presenting or developing jaundice early in their liver injury deserve close follow-up and perhaps early consultation with a transplant center, particularly if the injury pattern is hepatocellular.

For those that do not have an early severe outcome, the course is less clear although most patients are expected to have a full recovery. Indeed, DILI has typically been considered an outcome of extremes from early mortality or need for transplantation on one end and complete recovery on the other. However, even as early as 1999, a study of just 33 patients suggested that chronic damage on biopsy may occur during prolonged follow-up. ${ }^{82}$ Case reports of vanishing bile duct syndrome after a DILI episode are also reported. More recent registry data suggest chronic liver injury does occur, but the reported rate is highly dependent on how it is defined. At this point there are no accepted definitions for "chronic 
Table 4 Roussel Uclaf causality assessment model (RUCAM) causality assessment method ${ }^{37}$

\begin{tabular}{|c|c|c|c|c|c|c|}
\hline \multirow{3}{*}{$\begin{array}{l}\text { Criteria } \\
\text { Enzyme pattern } \\
\text { Exposure }\end{array}$} & \multicolumn{6}{|c|}{ RUCAM } \\
\hline & \multicolumn{3}{|l|}{ Hepatocellular } & \multicolumn{3}{|c|}{ Cholestatic or mixed } \\
\hline & Initial exposure & Subsequent exposure & Pts & Initial exposure & Subsequent exposure & Pts \\
\hline Timing from & 5-90 days & $1-15$ days & +2 & 5-90 days & $1-90$ days & +2 \\
\hline Drug start & $<5,>90$ days & $>15$ days & +1 & $<5,>90$ days & $>90$ days & +1 \\
\hline Drug stop & $\leq 15$ days & $\leq 15$ days & +1 & $\leq 30$ days & $\leq 30$ days & +1 \\
\hline Course & \multicolumn{3}{|c|}{$\begin{array}{l}\text { Difference between peak ALT and upper } \\
\text { limit normal (ULN) value }\end{array}$} & \multicolumn{3}{|c|}{$\begin{array}{l}\text { Difference between peak AP (or bili) and upper } \\
\text { limit normal (ULN) }\end{array}$} \\
\hline \multirow[t]{4}{*}{ After drug stop } & \multicolumn{2}{|c|}{ Decrease $\geq 50 \%$ in 8 days } & +3 & \multicolumn{2}{|c|}{ Decrease $\geq 50 \%$ in 180 days } & +2 \\
\hline & \multicolumn{2}{|c|}{ Decrease $\geq 50 \%$ in 30 days } & +2 & \multicolumn{2}{|c|}{ Decrease $<50 \%$ in 180 days } & +1 \\
\hline & \multicolumn{2}{|c|}{ Decrease $\geq 50 \%$ in $>30$ days } & 0 & \multicolumn{2}{|c|}{ Persistence or increase or no information } & 0 \\
\hline & \multicolumn{2}{|c|}{ Decrease $<50 \%$ in $>30$ days } & -2 & & & \\
\hline \multirow[t]{2}{*}{ Risk factor } & \multicolumn{2}{|l|}{ Ethanol: yes } & +1 & \multicolumn{2}{|c|}{ Ethanol or pregnancy: yes } & +1 \\
\hline & \multicolumn{2}{|l|}{ Ethanol: no } & 0 & \multicolumn{2}{|c|}{ Ethanol or pregnancy: no } & 0 \\
\hline \multirow[t]{2}{*}{ Age } & \multicolumn{2}{|l|}{$\geq 55$} & +1 & \multicolumn{2}{|l|}{$\geq 55$} & +1 \\
\hline & \multicolumn{2}{|l|}{$<55$} & 0 & \multicolumn{2}{|l|}{$<55$} & 0 \\
\hline \multirow[t]{4}{*}{ Other drugs } & \multicolumn{2}{|c|}{ None or no information } & 0 & \multicolumn{2}{|c|}{ None or no information } & 0 \\
\hline & \multicolumn{2}{|c|}{ Drug with suggestive timing } & -1 & \multicolumn{2}{|c|}{ Drug with suggestive timing } & -1 \\
\hline & \multicolumn{2}{|c|}{ Known hepatotoxin w/ suggestive timing } & -2 & Known hepatoto & $\mathrm{n} \mathrm{w} /$ suggestive timing & -2 \\
\hline & $\begin{array}{l}\text { Drug w/ other e } \\
\text { (e.g., + rechalle }\end{array}$ & $\begin{array}{l}\text { dence for a role } \\
\text { e) }\end{array}$ & -3 & $\begin{array}{l}\text { Drug w/ other e } \\
\text { (e.g., + rechalle }\end{array}$ & $\begin{array}{l}\text { dence for a role } \\
\text { e) }\end{array}$ & -3 \\
\hline Competing causes & & & & & & \\
\hline & All Group $\left.\right|^{\mathrm{a}} \& \mid I^{\mathrm{b}}$ & uled out & +2 & All Group $\left.\right|^{\mathrm{a}} \&\|\|^{\mathrm{b}}$ & uled out & +2 \\
\hline & All of Group I ru & out & +1 & All of Group I ru & d out & +1 \\
\hline & 4-5 of Group I r & ed out & 0 & 4-5 of Group I r & ed out & 0 \\
\hline & $<4$ of Group I ru & $\mathrm{d}$ out & -2 & $<4$ of Group I rL & $\mathrm{d}$ out & -2 \\
\hline & Nondrug cause & hly probable & -3 & Nondrug cause & ghly probable & -3 \\
\hline Previous information & Reaction in prod & t label & +2 & Reaction in prod & ct label & +2 \\
\hline & Reaction publish & ; no label & +1 & Reaction publish & ; no label & +1 \\
\hline & Reaction unknov & & 0 & Reaction unknov & & 0 \\
\hline Rechallenge & Positive & & +3 & Positive & & +3 \\
\hline & Compatible & & +1 & Compatible & & +1 \\
\hline & Negative & & -2 & Negative & & -2 \\
\hline & Not done or not & terpretable & 0 & Not done or not & terpretable & 0 \\
\hline
\end{tabular}

${ }^{a}$ Group I, HAV, HBV, HCV (acute), biliary obstruction, alcoholism, recent hypotension (shock liver).

${ }^{\mathrm{b}}$ Group II, CMV, EBV, herpes virus infection.

DILI," hence the literature in this area is unclear but evolving. In the Sweden registry, 685 patients surviving the first few months after their DILI episode were linked to their national Cause of Death Registry and Hospital Discharge Registry. ${ }^{83}$ Follow-up spanned a remarkable median of 11 years (range 3-23). Twenty-three patients (3.4\%) were diagnosed with liver disease during a hospitalization or at death, and medical charts reviewed. Of these 23, perhaps 10 (1.4\%) had chronic DILI based on chart review indicating no other obvious etiology for their liver disorder. Such criteria based on hospitalization and/or death registration will obviously underesti- mate the rate by excluding those with less severe course and followed in an outpatient setting.

When chronic DILI is defined more broadly as persistent elevations in liver enzymes, the rate is expectedly higher. In the Spanish registry, chronic DILI was defined as persistently elevated liver biochemistries at 3 months post-DILI for hepatocellular and 6 months for cholestatic or mixed injuries. Here, the overall rate of chronicity was $5.7 \%$. In the U.S. DILIN registry, $18 \%$ had persistent elevations at 6 months including all patterns of injury. ${ }^{84}$ The clinical and histologic outcome of such patients remains unclear. Moreover, subsequent 
development of non-DILI liver disease such nonalcoholic fatty liver disease and prolonged resolution of biochemistries beyond 6 months will need to be considered moving forward. Nevertheless, data suggest chronic DILI whether by immunemediated injury, vanishing bile ducts, or some other pathophysiology, does exist and may portend future liver-related problems for some.

Thus, the natural history of DILI is dominated by complete recovery for most, but roughly $10 \%$ may not survive the initial injury or may require liver transplantation. Another 5 to $10 \%$ may be at risk for chronic injury and perhaps long-term morbidity and mortality. The role of clinical cofactors (e.g., NAFLD) and comorbidities (e.g., diabetes) in the risk of developing chronic DILI requires further investigation. Clearly, heterogeneity in hepatotoxic agents and host susceptibility factors play important roles in both early severe outcome and chronic injury. For now, the clinician should be aware of the medications, signs, and symptoms indicating increased risk of early severe outcome. And for the majority of patients surviving the initial injury, the clinician must remember that some may not fully resolve and deserve follow-up.

\section{Conclusion}

The clinical manifestations, diagnosis, and natural history of idiosyncratic DILI remain a challenge for the busy gastroenterologist. Despite its low incidence in the general population, ${ }^{3,4}$ DILI remains a common request for gastroenterology consultation both in the inpatient and outpatient setting. Without objective diagnostic tests, clinicians must rely heavily on history taking skills, awareness of the hepatotoxicity risk for various agents, in-depth knowledge of clinical presentation, and evaluation for competing etiologies. Occasionally, DILI may be severe or life-threatening, and risks factors for such must be recognized quickly to provide appropriate care. Thereafter, a minimum of 6 months follow-up to assess for possible chronic injury is necessary. All this takes time, which can be at a premium for the busy clinician. Better diagnostic tools and epidemiologic data will make DILI identification and care easier in the future. For now, the clinician may want to keep the RUCAM ( - Table 4$)^{37}$ or a clinical checklist $(\mathbf{- T a b l e ~ 2})^{40}$ close at hand and refer to the LiverTox website for guidance on particular agents.

\section{References}

1 Chalasani N, Fontana RJ, Bonkovsky HL, et al; Drug Induced Liver Injury Network (DILIN). Causes, clinical features, and outcomes from a prospective study of drug-induced liver injury in the United States. Gastroenterology 2008;135(6):1924-1934, e1-e4

2 Andrade RJ, Lucena MI, Fernández MC, et al; Spanish Group for the Study of Drug-Induced Liver Disease. Drug-induced liver injury: an analysis of 461 incidences submitted to the Spanish registry over a 10-year period. Gastroenterology 2005;129(2):512-521

3 Björnsson ES, Bergmann OM, Björnsson HK, Kvaran RB, Olafsson S. Incidence, presentation, and outcomes in patients with druginduced liver injury in the general population of Iceland. Gastroenterology 2013;144(7):1419-1425, e1-e3, quiz e19-e20
4 Sgro C, Clinard F, Ouazir K, et al. Incidence of drug-induced hepatic injuries: a French population-based study. Hepatology 2002; 36(2):451-455

5 Ostapowicz G, Fontana RJ, Schiødt FV, et al; U.S. Acute Liver Failure Study Group. Results of a prospective study of acute liver failure at 17 tertiary care centers in the United States. Ann Intern Med 2002; 137(12):947-954

6 Watkins PB. Drug safety sciences and the bottleneck in drug development. Clin Pharmacol Ther 2011;89(6):788-790

7 Temple R. Hy's law: predicting serious hepatotoxicity. Pharmacoepidemiol Drug Saf 2006;15(4):241-243

8 Björnsson E, Olsson R. Outcome and prognostic markers in severe drug-induced liver disease. Hepatology 2005;42(2):481-489

9 Polson J, Lee WM; American Association for the Study of Liver Disease. AASLD position paper: the management of acute liver failure. Hepatology 2005;41(5):1179-1197

10 Lee WM. Drug-induced acute liver failure. Clin Liver Dis 2013; 17(4):575-586, viii

11 Mindikoglu AL, Magder LS, Regev A. Outcome of liver transplantation for drug-induced acute liver failure in the United States: analysis of the United Network for Organ Sharing database. Liver Transpl 2009;15(7):719-729

12 Danielides IC, Constantoulakis M, Daikos GK. Hepatitis on high dose isoniazid: reintroduction of the drug in severe tuberculous meningitis. Am J Gastroenterol 1983;78(6):378-380

13 LiverTox. Isoniazid. Available at: http://livertox.nlm.nih.gov/Isoniazid.htm. Accessed June 6, 2013

14 Black M, Mitchell JR, Zimmerman HJ, Ishak KG, Epler GR. Isoniazidassociated hepatitis in 114 patients. Gastroenterology 1975;69(2): 289-302

15 Harrill AH, Roach J, Fier I, et al. The effects of heparins on the liver: application of mechanistic serum biomarkers in a randomized study in healthy volunteers. Clin Pharmacol Ther 2012;92(2): 214-220

16 LiverTox. Heparins. Available at: http://livertox.nlm.nih.gov/Heparin.htm. Accessed October 4, 2013

17 McDonald C, Uy J, Hu W, et al. Clinical significance of hyperbilirubinemia among HIV-1-infected patients treated with atazanavir/ritonavir through 96 weeks in the CASTLE study. AIDS Patient Care STDS 2012;26(5):259-264

18 Grant L, Lee WL, Rockey DC. Trimethoprim/Sulfamethoxazole hepatotoxicity: Analysis of 31 cases. Hepatology 2013; 58(4, Suppl)382A

19 Blumenthal KG, Patil SU, Long AA. The importance of vancomycin in drug rash with eosinophilia and systemic symptoms (DRESS) syndrome. Allergy Asthma Proc 2012;33(2):165-171

20 Fleming P, Marik PE. The DRESS syndrome: the great clinical mimicker. Pharmacotherapy 2011;31(3):332

21 Björnsson E. Hepatotoxicity associated with antiepileptic drugs. Acta Neurol Scand 2008;118(5):281-290

22 Björnsson E, Talwalkar J, Treeprasertsuk S, et al. Drug-induced autoimmune hepatitis: clinical characteristics and prognosis. Hepatology 2010;51(6):2040-2048

23 Ghabril M, Bonkovsky HL, Kum C, et al; US Drug-Induced Liver Injury Network. Liver injury from tumor necrosis factor- $\alpha$ antagonists: analysis of thirty-four cases. Clin Gastroenterol Hepatol 2013;11(5):558, e3

24 Zimmerman HJ. Oncotherapeutic and immunosuppressive agents. In: Zimmerman HJ. Hepatotoxicity: the adverse effects of drugs and other chemicals on the liver. 2nd ed. Philadelphia: Lippincott Williams \& Wilkins; 1999:681-687

25 Kalb RE, Strober B, Weinstein G, Lebwohl M. Methotrexate and psoriasis: 2009 National Psoriasis Foundation Consensus Conference. J Am Acad Dermatol 2009;60(5):824-837

26 Saag KG, Teng GG, Patkar NM, et al; American College of Rheumatology. American College of Rheumatology 2008 recommendations for the use of nonbiologic and biologic disease-modifying 
antirheumatic drugs in rheumatoid arthritis. Arthritis Rheum 2008;59(6):762-784

27 Kremer JM, Alarcón GS, Lightfoot RW Jr, et al; American College of Rheumatology. Methotrexate for rheumatoid arthritis. Suggested guidelines for monitoring liver toxicity. Arthritis Rheum 1994; 37(3):316-328

28 LiverTox. Valproate. Available at: http://livertox.nlm.nih.gov/Valproate.htm. Accessed November 5, 2013

29 Zimmerman HJ. Psychotropic and anticonvulsant agents. In: Zimmerman HJ. Hepatotoxicity: the adverse effects of drugs and other chemicals on the liver. 2nd ed. Philadelphia: Lippincott Williams \& Wilkins; 1999:504-508

30 Dealberto MJ. Valproate-induced hyperammonaemic encephalopathy: review of 14 cases in the psychiatric setting. Int Clin Psychopharmacol 2007;22(6):330-337

31 Uusimaa J, Hinttala R, Rantala H, et al. Homozygous W748S mutation in the POLG1 gene in patients with juvenile-onset Alpers syndrome and status epilepticus. Epilepsia 2008;49(6): 1038-1045

32 Angitapalli R, Litwin AM, Kumar PR, et al. Adjuvant FOLFOX chemotherapy and splenomegaly in patients with stages II-III colorectal cancer. Oncology 2009;76(5):363-368

33 Slade JH, Alattar ML, Fogelman DR, et al. Portal hypertension associated with oxaliplatin administration: clinical manifestations of hepatic sinusoidal injury. Clin Colorectal Cancer 2009;8(4): 225-230

34 DeLeve LD, Valla DC, Garcia-Tsao G; American Association for the Study Liver Diseases. Vascular disorders of the liver. Hepatology 2009;49(5):1729-1764

35 McDonald GB, Hinds MS, Fisher LD, et al. Veno-occlusive disease of the liver and multiorgan failure after bone marrow transplantation: a cohort study of 355 patients. Ann Intern Med 1993;118(4): 255-267

36 Jones RJ, Lee KS, Beschorner WE, et al. Venoocclusive disease of the liver following bone marrow transplantation. Transplantation 1987;44(6):778-783

37 Danan G, Benichou C. Causality assessment of adverse reactions to drugs-I. A novel method based on the conclusions of international consensus meetings: application to drug-induced liver injuries. J Clin Epidemiol 1993;46(11):1323-1330

38 Benichou C, Danan G, Flahault A. Causality assessment of adverse reactions to drugs-II. An original model for validation of drug causality assessment methods: case reports with positive rechallenge. J Clin Epidemiol 1993;46(11):1331-1336

39 Maria VA, Victorino RM. Development and validation of a clinical scale for the diagnosis of drug-induced hepatitis. Hepatology 1997;26(3):664-669

40 Agarwal VK, McHutchison JG, Hoofnagle JH; Drug-Induced Liver Injury Network. Important elements for the diagnosis of druginduced liver injury. Clin Gastroenterol Hepatol 2010;8(5): 463-470

41 Fontana RJ, Seeff LB, Andrade RJ, et al. Standardization of nomenclature and causality assessment in drug-induced liver injury: summary of a clinical research workshop. Hepatology 2010;52(2): 730-742

42 Barritt AS IV, Lee J, Hayashi PH. Detective work in drug-induced liver injury: sometimes it is all about interviewing the right witness. Clin Gastroenterol Hepatol 2010;8(7):635-637

43 Bailey RL, Gahche JJ, Lentino CV, et al. Dietary supplement use in the United States, 2003-2006. J Nutr 2011;141(2):261-266

44 Navarro VJ, Barnhart HX, Bonkovsky HL, et al. The rising burden of herbal and dietary supplement induced hepatotoxicity in the U.S. A. Hepatology 2013;58(4, suppl):264A

45 Navarro VJ, Bonkovsky HL, Hwang SI, Vega M, Barnhart H, Serrano J. Catechins in dietary supplements and hepatotoxicity. Dig Dis Sci 2013;58(9):2682-2690

46 Means B, Swan GE, Jobe JB, Esposito JL. An alternative approach to obtaining personal history data. In: Biemeer PP, Groves RM, Lyberg
LE, Mathiowetz NA, Sudman S, eds. Measurement of Errors in Surveys. New York: John Wiley and Sons; 1991:167-183

47 Friedenreich CM. Improving long-term recall in epidemiologic studies. Epidemiology 1994;5(1):1-4

48 Bénichou C. Criteria of drug-induced liver disorders. Report of an international consensus meeting. J Hepatol 1990;11(2): 272-276

49 Lammert C, Einarsson S, Saha C, Niklasson A, Bjornsson E, Chalasani N. Relationship between daily dose of oral medications and idiosyncratic drug-induced liver injury: search for signals. Hepatology 2008;47(6):2003-2009

50 Chen M, Borlak J, Tong W. High lipophilicity and high daily dose of oral medications are associated with significant risk for druginduced liver injury. Hepatology 2013;58(1):388-396

51 Food and Drug Administration. Summary of NDA Approvals \& Receipts, 1938 to the present. Available at: http://www.fda.gov/ AboutFDA/WhatWeDo/History/ProductRegulation/SummaryofNDAApprovalsReceipts1938tothepresent/default.htm. Accessed March 31, 2013

52 National Library of Medicine. LiverTox. Available at: http://www. LiverTox.nih.gov. Accessed March 31, 2013

53 Hoofnagle JH, Serrano J, Knoben JE, Navarro VJ. LiverTox: a website on drug-induced liver injury. Hepatology 2013;57(3):873-874

54 Yeoman AD, Westbrook RH, Al-Chalabi T, et al. Diagnostic value and utility of the simplified International Autoimmune Hepatitis Group (IAIHG) criteria in acute and chronic liver disease. Hepatology 2009;50(2):538-545

55 Fernández-Ruiz M, Muñoz-Codoceo C, López-Medrano F, et al. Cytomegalovirus myopericarditis and hepatitis in an immunocompetent adult: successful treatment with oral valganciclovir. Intern Med 2008;47(22):1963-1966

56 Cohen JI, Corey GR. Cytomegalovirus infection in the normal host. Medicine (Baltimore) 1985;64(2):100-114

57 Gupta E, Bhatia V, Choudhary A, Rastogi A, Gupta NL. Epstein-Barr virus associated acute hepatitis with cross-reacting antibodies to other herpes viruses in immunocompetent patients: report of two cases. J Med Virol 2013;85(3):519-523

58 Riviere Marcelin J, Jones JM, Costello BA. 41-year-old woman with fever, neutropenia, and elevated transaminase levels. Mayo Clin Proc 2013;88(1):113-116

59 Ichai P, Roque Afonso AM, Sebagh M, et al. Herpes simplex virusassociated acute liver failure: a difficult diagnosis with a poor prognosis. Liver Transpl 2005;11(12):1550-1555

60 Dalton HR, Fellows HJ, Stableforth W, et al. The role of hepatitis E virus testing in drug-induced liver injury. Aliment Pharmacol Ther 2007;26(10):1429-1435

61 Davern TJ, Chalasani N, Fontana RJ, et al; Drug-Induced Liver Injury Network (DILIN).Acute hepatitis E infection accounts for some cases of suspected drug-induced liver injury. Gastroenterology 2011;141(5):1665-1672, e1-e9

62 Hoofnagle JH, Nelson KE, Purcell RH. Hepatitis E. N Engl J Med 2012;367(13):1237-1244

63 European Association for Study of Liver. EASL Clinical Practice Guidelines: Wilson's disease. J Hepatol 2012;56(3):671-685

64 Dowling D. Screening for Wilson's disease in acute liver failure: a comparison of currently available diagnostic tests. Hepatology 2009;50(1):329

65 Maple JT, Ben-Menachem T, Anderson MA, et al; ASGE Standards of Practice Committee. The role of endoscopy in the evaluation of suspected choledocholithiasis. Gastrointest Endosc 2010;71(1): $1-9$

66 Yang $\mathrm{MH}$, Chen TH, Wang SE, et al. Biochemical predictors for absence of common bile duct stones in patients undergoing laparoscopic cholecystectomy. Surg Endosc 2008;22(7):16201624

67 Lindor KD, Gershwin ME, Poupon R, Kaplan M, Bergasa NV, Heathcote EJ; American Association for Study of Liver Diseases. Primary biliary cirrhosis. Hepatology 2009;50(1):291-308 
68 Chapman R, Fevery J, Kalloo A, et al; American Association for the Study of Liver Diseases. Diagnosis and management of primary sclerosing cholangitis. Hepatology 2010;51(2):660-678

69 Farrell GC. Drug-Induced Liver Disease. Edinburgh, UK: Churchill Livingstone; 1994

70 Lewis JH, Kleiner DE. Hepatic injury due to drugs, chemicals and toxins. In: Burt AD, Portmann BC, Ferrell LD, eds. MacSween's Pathology of the Liver. Philadelphia, PA: Elsevier; 2007

71 Kleiner DE, Chalasani NP, Lee WM, et al; Drug-Induced Liver Injury Network (DILIN). Hepatic histological findings in suspected druginduced liver injury: systematic evaluation and clinical associations. Hepatology 2014;59(2):661-670

72 Björnsson E, Kalaitzakis E, Olsson R. The impact of eosinophilia and hepatic necrosis on prognosis in patients with drug-induced liver injury. Aliment Pharmacol Ther 2007;25(12):1411-1421

73 Katoonizadeh A, Nevens F, Verslype C, Pirenne J, Roskams T. Liver regeneration in acute severe liver impairment: a clinicopathological correlation study. Liver Int 2006;26(10):1225-1233

74 Hennes EM, Zeniya M, Czaja AJ, et al; International Autoimmune Hepatitis Group. Simplified criteria for the diagnosis of autoimmune hepatitis. Hepatology 2008;48(1):169-176

75 Czaja AJ. Corticosteroids or not in severe acute or fulminant autoimmune hepatitis: therapeutic brinksmanship and the point beyond salvation. Liver Transpl 2007;13(7):953-955

76 Ichai P, Duclos-Vallée JC, Guettier C, et al. Usefulness of corticosteroids for the treatment of severe and fulminant forms of autoimmune hepatitis. Liver Transpl 2007;13(7):996-1003
77 Berends MA, van Oijen MG, Snoek J, et al. Reliability of the Roenigk classification of liver damage after methotrexate treatment for psoriasis: a clinicopathologic study of 160 liver biopsy specimens. Arch Dermatol 2007;143(12):1515-1519

78 Rochon J, Protiva P, Seeff LB, et al; Drug-Induced Liver Injury Network (DILIN). Reliability of the Roussel Uclaf Causality Assessment Method for assessing causality in drug-induced liver injury. Hepatology 2008;48(4):1175-1183

79 Rockey DC, Seeff LB, Rochon J, et al; US Drug-Induced Liver Injury Network. Causality assessment in drug-induced liver injury using a structured expert opinion process: comparison to the RousselUclaf causality assessment method. Hepatology 2010;51(6): 2117-2126

80 Fontana RJ, Watkins PB, Bonkovsky HL, et al; DILIN Study Group. Drug-Induced Liver Injury Network (DILIN) prospective study: rationale, design and conduct. Drug Saf 2009;32(1):55-68

81 Kaplowitz N. Causality assessment versus guilt-by-association in drug hepatotoxicity. Hepatology 2001;33(1):308-310

82 Aithal PG, Day CP. The natural history of histologically proved drug induced liver disease. Gut 1999;44(5):731-735

83 Björnsson E, Davidsdottir L. The long-term follow-up after idiosyncratic drug-induced liver injury with jaundice. J Hepatol 2009; 50(3):511-517

84 Fontana RJ, Hayashi PH, Gu J, et al. Idiosyncratic drug induced liver injury is associated with substantial morbidity and mortality within 6 months from onset.. Gastroenterology 2014;56; Mar 26 [Epub ahead of print] 